\title{
Defining Relations of a Free Modular Lattice of Rank 3
}

\author{
A. G. Gein* and M. P. Shushpanov** \\ (Submitted by L.N. Shevrin) \\ Ural Federal University, ul. Mira 19, Ekaterinburg, 620002 Russia \\ Received February 11, 2013
}

\begin{abstract}
For a 3-generated free modular lattice we obtain a set of 11 defining relations and prove that this set is minimal.
\end{abstract}

DOI: $10.3103 / \mathrm{S} 1066369 \mathrm{X} 13100071$

Keywords and phrases: free modular lattices, defining relations.

Recall that the rank of free algebra from some manifold is the cardinal number of the set of its free generators. We concentrate our attention on a free lattice of the rank 3 in the manifold of modular lattices; we denote it by $A$. Let $F$ be a free lattice of the rank 3 in the manifold of all lattices; let $f, g$, and $h$ be its free generators, and let $\varphi$ be a homomorphism from $F$ to $A$. By standard considerations of a universal algebra, elements $a=\varphi(f), b=\varphi(g)$, and $c=\varphi(h)$ are free generators of the lattice $A$. Relations defining this lattice in the manifold of all lattices were considered in [1] and [2]. In the paper [1] one has particularly shown that $A$ can be defined by 21 relations. In [2] one has proved that this set of defining relations is not minimal; namely, it was shown there that 15 relations among those mentioned above define the lattice $A$, moreover, they form the minimal set of defining relations for $A$. Note that in [1] one has described a set of seven defining relations for a free distributive lattice of the rank 3 , and in [3] this set was proved to be minimal.

The following assertion is the main result of this paper: There exists a set of 11 defining relations for the lattice $A$. Note that this set is not a subset of the set of defining relations indicated in [1]. Let us enumerate these relations:

$$
\begin{aligned}
& (a \vee(b \wedge c)) \wedge(b \vee c)=(a \wedge(b \vee c)) \vee(b \wedge c) \\
& (b \vee(c \wedge a)) \wedge(c \vee a)=(b \wedge(c \vee a)) \vee(c \wedge a), \\
& (c \vee(a \wedge b)) \wedge(a \vee b)=(c \wedge(a \vee b)) \vee(a \wedge b),
\end{aligned}
$$

$$
\begin{aligned}
(a \vee b) \wedge(a \vee c) \wedge(b \vee c)=( & (a \\
& \wedge(b \vee c)) \vee((a \vee b) \wedge c)) \\
& \wedge((b \wedge(a \vee c)) \vee((b \vee a) \wedge c)) \wedge((a \wedge(c \vee b)) \vee((a \vee c) \wedge b)),
\end{aligned}
$$

$(a \wedge b) \vee(a \wedge c) \vee(b \wedge c)=((a \vee(b \wedge c)) \wedge((a \wedge b) \vee c))$

$$
\vee((b \vee(a \wedge c)) \wedge((b \wedge a) \vee c)) \vee((a \vee(c \wedge b)) \wedge((a \wedge c) \vee b)),
$$

$(a \vee b) \wedge(a \vee c)=a \vee((a \vee b) \wedge(a \vee c) \wedge(b \vee c))$

$(b \vee a) \wedge(b \vee c)=b \vee((a \vee b) \wedge(a \vee c) \wedge(b \vee c))$

$(c \vee a) \wedge(c \vee b)=c \vee((a \vee b) \wedge(a \vee c) \wedge(b \vee c))$,

$(a \wedge b) \vee(a \wedge c)=a \wedge((a \wedge b) \vee(a \wedge c) \vee(b \wedge c))$,

$$
(b \wedge a) \vee(b \wedge c)=b \wedge((a \wedge b) \vee(a \wedge c) \vee(b \wedge c))
$$

\footnotetext{
*E-mail: Alexander.Gein@usu.ru.

${ }^{* *}$ E-mail: Mikhail.Shushpanov@gmail.com.
} 


$$
(c \wedge a) \vee(c \wedge b)=c \wedge((a \wedge b) \vee(a \wedge c) \vee(b \wedge c))
$$

For short we denote this set of relations by $\rho$.

Theorem. The lattice with generating elements $a, b$, and $c$ and with the set $\rho$ of defining relations is isomorphic to a free modular lattice of the rank 3. If a subset of relations is strictly contained in $\rho$, then the lattice with generating elements $a, b$, and $c$ and with this set of defining relations is not modular.

The proof of the first statement of the theorem is based on the following assertions. Let $L$ be the lattice with generating elements $a, b$, and $c$ defined by relations of the set $\rho$. Since the lattice $A$ satisfies the indicated relations, it is a homomorphic image of the lattice $L$. It is known that $A$ contains 28 elements, therefore, the lattice $L$ contains at least 28 elements. But direct calculations show that $L$ cannot contain more than 28 elements. This means that two mentioned lattices are isomorphic.

Let us prove the second assertion. For each set of defining relations obtained from $\rho$ by eliminating one arbitrary relation we construct an example of a 3-generated non-modular lattice satisfying all relations from the mentioned set. For instance, the lattice presented in Fig. 1 is non-modular; it satisfies relations (2)-(11) but does not satisfy relation (1).

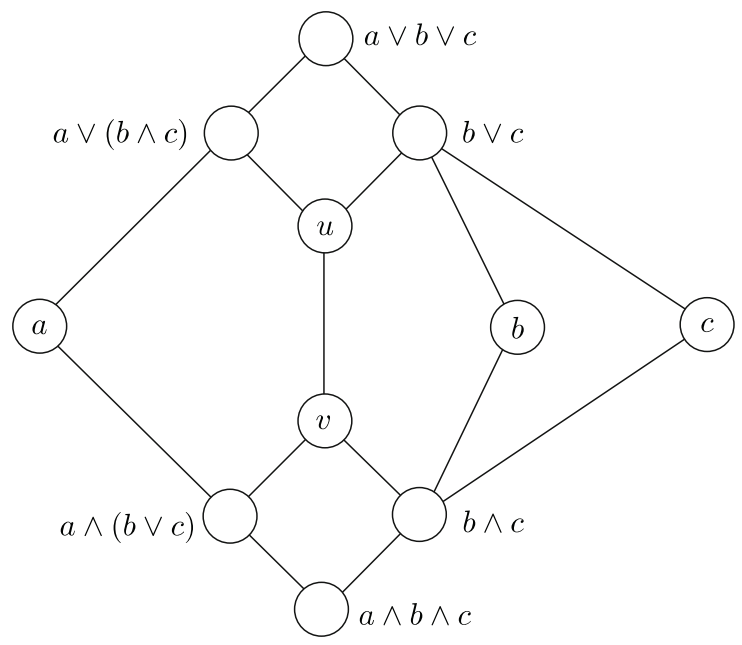

Fig. 1.

In Fig. 1 we use the denotations $u=(a \vee(b \wedge c)) \wedge(b \vee c)$ and $v=(a \wedge(b \vee c)) \vee(b \wedge c)$.

The lattice presented in Fig. 2 satisfies relations (1)-(3) and (5)-(11) but does not satisfy relation (4).

In Fig. 2 we use the denotations $t=(a \vee b) \wedge(a \vee c) \wedge(b \vee c), a_{1}=a \wedge(b \vee c)$, and $b_{1}=b \wedge(a \vee c)$.

The lattice presented in Fig. 3 satisfies relations (1)-(5) and (7)-(11) but does not satisfy relation (6).

The rest examples are obtained from those described above by permutations of elements $a, b$, and $c$ and by passing to the dual lattices.

In this connection it is interesting to clear out whether there exists a set of defining relations for a free modular lattice of the rank 3 , where the number of relations is less than 11 .

\section{ACKNOWLEDGMENTS}

We are grateful to the reviewer for pointing out our attention to the paper [2], owing to which we have essentially revised our paper. We also express gratitude to L. N. Shevrin for useful discussions. 


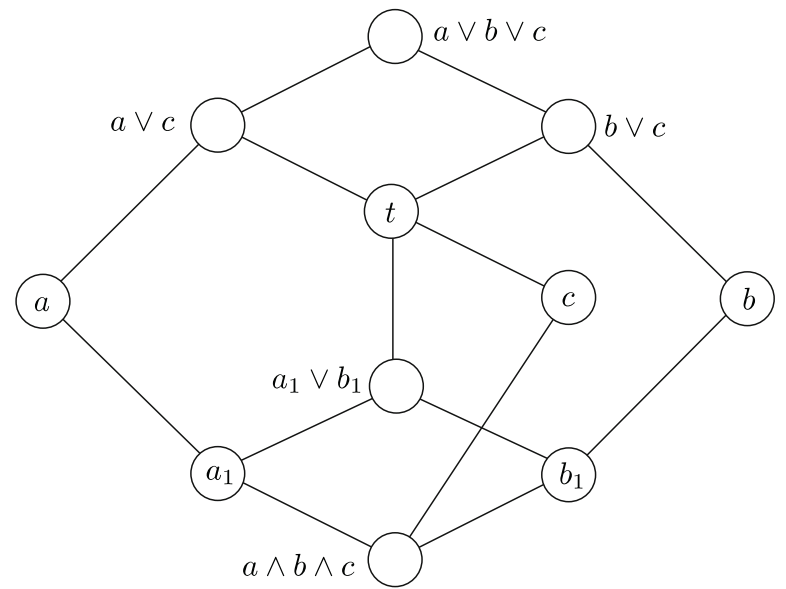

Fig. 2.

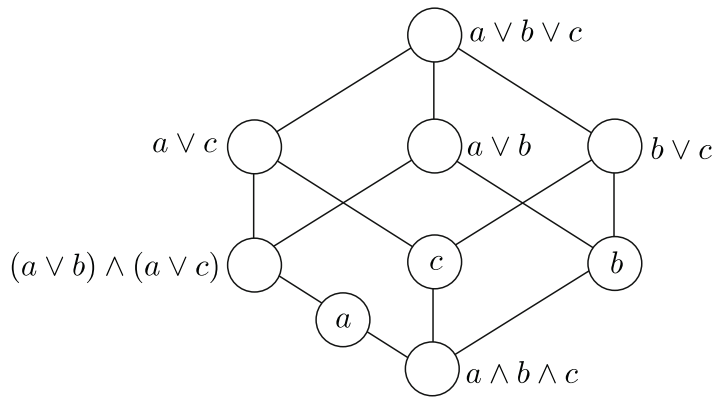

Fig. 3.

\section{REFERENCES}

1. O. Ore, "Remarks on Structures and Group Relations," Vierteljahrsschrift der Naturforschenden Gesellschaft in Zurich 85 (32), 1-4 (1940).

2. Z. Ladzianska, "Modular Sublattices of a Lattice," Mat. časop. 24 (1), 81-83 (1974).

3. M. Kolibiar, "Distributive Sublattices of a Lattice," Proc. Amer. Math. Soc. 34 (2), 359-364 (1972).

Translated by F. F. Sultanbekov 